\title{
Late Spontaneous Recanalization of Chronic Middle Cerebral Artery Occlusion
}

\author{
Cheol-Young Lee, MD', ${ }^{1,}$ Chang-Woo Ryu, MD², Jun-Seok Koh, MD', Gook Ki Kim, MD, PhD'
}

Early spontaneous recanalization of the middle cerebral artery in acute ischemic phase artery is not uncommon, whereas the late spontaneous recanalization of chronic occluded artery is a very rare phenomenon and exact incidence and the timing of this event have not been quantified. We present a case in which late spontaneous recanalization of long-lasting middle cerebral artery occlusion occurred in the absence of surgical, endovascular and thrombolytic treatments.

Key Words : Artheriosclerosis; Cerebrovascular disease; Angiography

Acute occlusion of middle cerebral artery (MCA) has been associated with high morbidity and mortality, compromising cerebral blood flow to the frontal and temporal lobes and the basal ganglia [1, 2]. However, chronic occlusion of MCA is sometimes identified incidentally by magnetic resonance (MR) angiography in asymptomatic patients or can present with ischemic stroke resulting from hemodynamic compromise. In chronic occlusion with enough collateral circulation, conservative methods including continued heparin and/or anti-platelet therapies can be used. Early spontaneous recanalization after acute embolic occlusion is a common phenomenon in the intracranial circulation. However, most observers agree that late spontaneous

${ }^{1}$ Department of Neurosurgery \& ${ }^{2}$ Radiology, Kyung Hee University Hospital at Gangdong, Seoul, Korea

${ }^{3}$ Department of Neurosurgery, Konyang University Hospital, Daejeon, Korea

Received November 5, 2011;

accepted after revision February 7, 2012.

Correspondence to: Cheol-Young Lee, MD, Department of Neurosurgery, Konyang University Hospital, 685, Gasoowon-dong, Seo-gu, Daejeon 302-718, Korea.

Tel. 82.42.600.9130 Fax. 82.42.600.9500

E-mail: leecy009@hanmail.net

This is an Open Access article distributed under the terms of the Creative Commons Attribution Non-Commercial License (http://creativecommons.org/licenses/by-nc/3.0) which permits unrestricted non-commercial use, distribution, and reproduction in any medium, provided the original work is properly cited. recanalization of the long-standing occlusion of intracranial artery is virtually impossible $[1,3]$. To our knowledge, late spontaneous recanalization of occluded artery has been documented only in the extracranial circulation. We report a case in which late spontaneous recanalization of chronic MCA occlusion occurred in the absence of surgical, endovascular and thrombolytic treatments. We found a limited documented report of late spontaneous recanalization of the posterior cerebral circulation [4]. To the best of our knowledge, this is the first published report for late spontaneous recanalization of the completely occluded MCA in chronic state.

\section{CASE REPORT}

A 53-year-old man presented with transient acute onset of left-sided numbness and speech disturbance one week prior to admission. These symptoms lasted for only several minutes and spontaneously resolved. Transient ischemic attack or acute cerebral infarction were possible diagnoses, so MRI and MR angiography were performed, showing an occlusion of his right MCA at the proximal M1 segment without any acute ischemic lesion or infracted area on MRI, including diffusion-weighted images. The next day, a conventional angiogram was taken and revealed a tapered occlusion at proximal M1 segment of right MCA, collateral pathways through perforating arteries, rich 


\section{Cheol-Young Lee, et al.}

pial collateralization from distal anterior cerebral artery, and a shift of the watershed zone (Fig. 1A, B). These findings were compatible with the long-standing stage of the occlusion of MCA rather than the acute occlusion. He also has an incidental saccular aneurysm at left posterior communicating artery (P-com) origin. The brain single photon emission computed tomography (SPECT) showed mildly decreased perfusion in
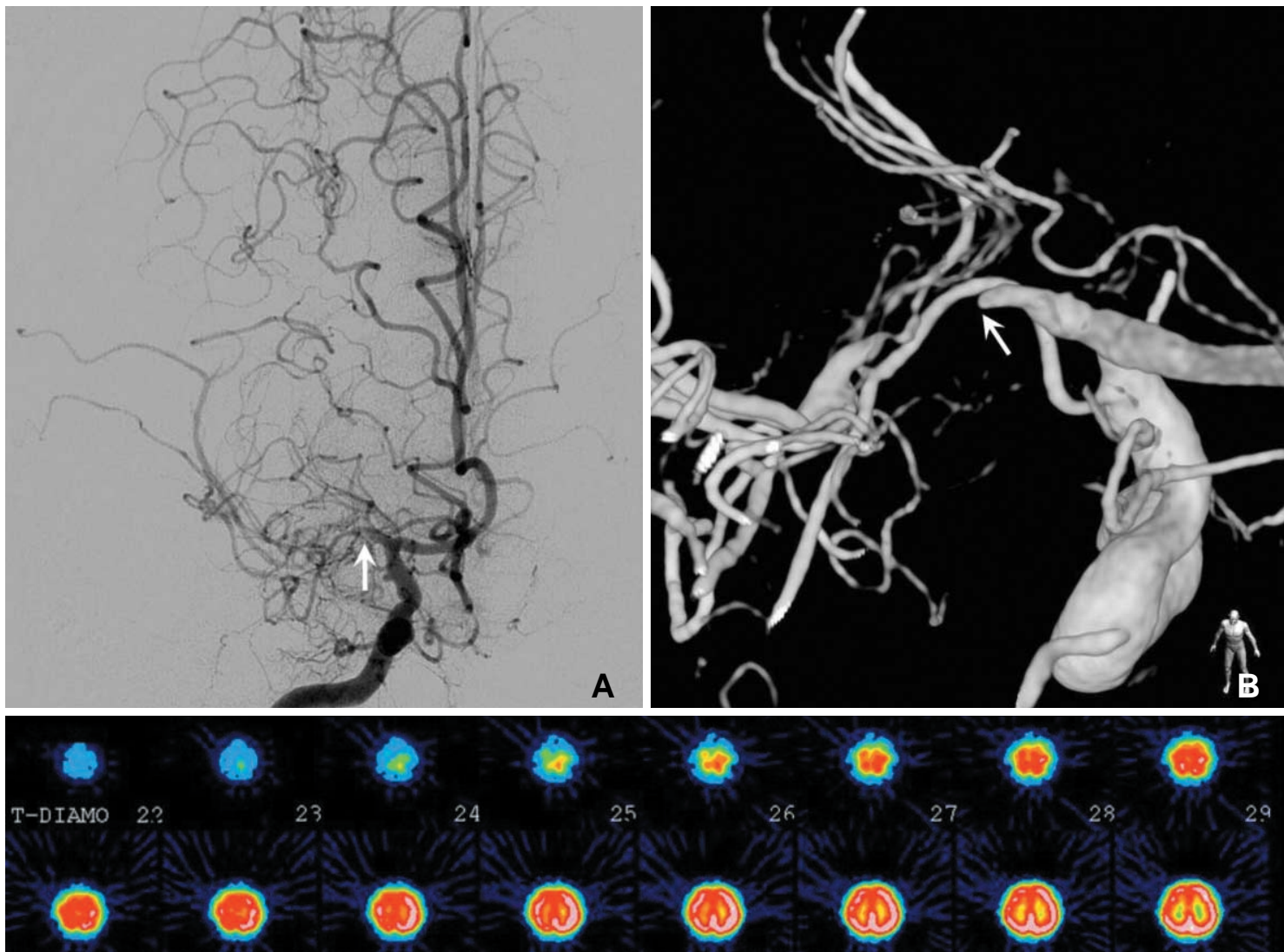

30
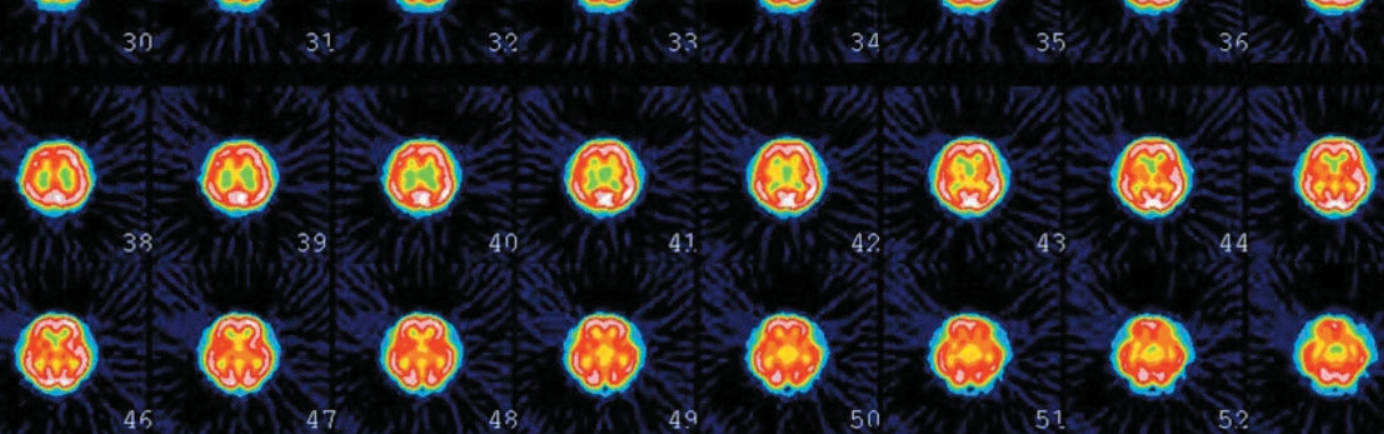
45
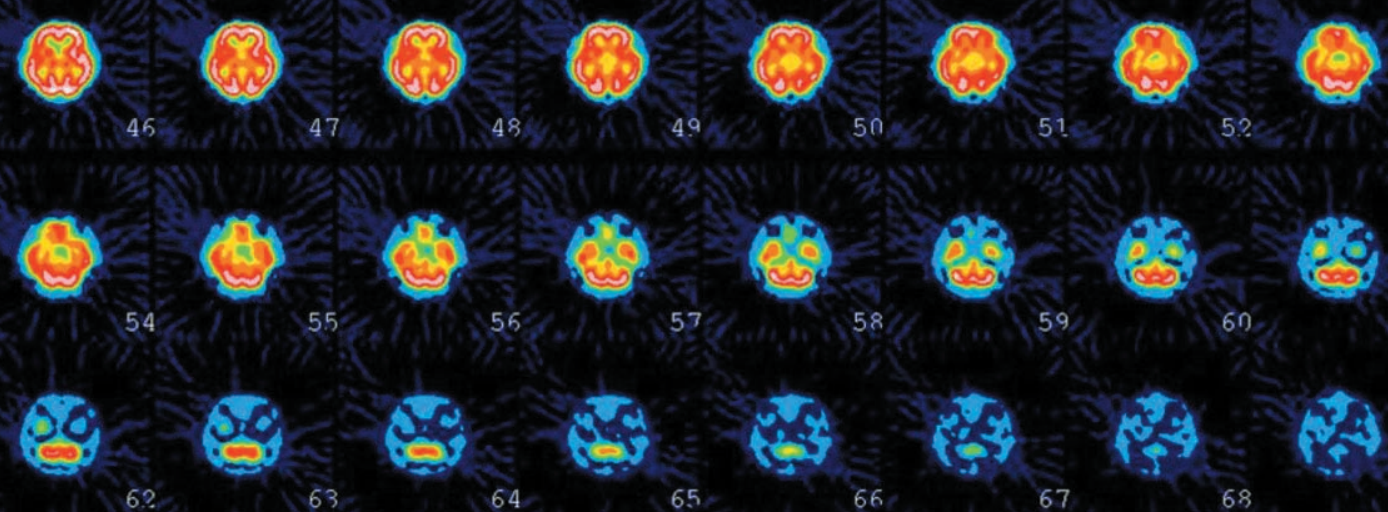

61

Fig. 1. A. An Initial right carotid angiogram shows the occlusion of the proximal trunk of right middle cerebral artery (MCA) (arrow). The pial collaterals from anterior cerebral artery and the shift of the watershed zone are noted.

B. Three-dimensional rotation angiography clearly reveals a tapered occlusion of proximal segment of M1 (arrow). Distal segment from occlusion and lenticulostriate arteries are filled by contrast media through basal collaterals. 


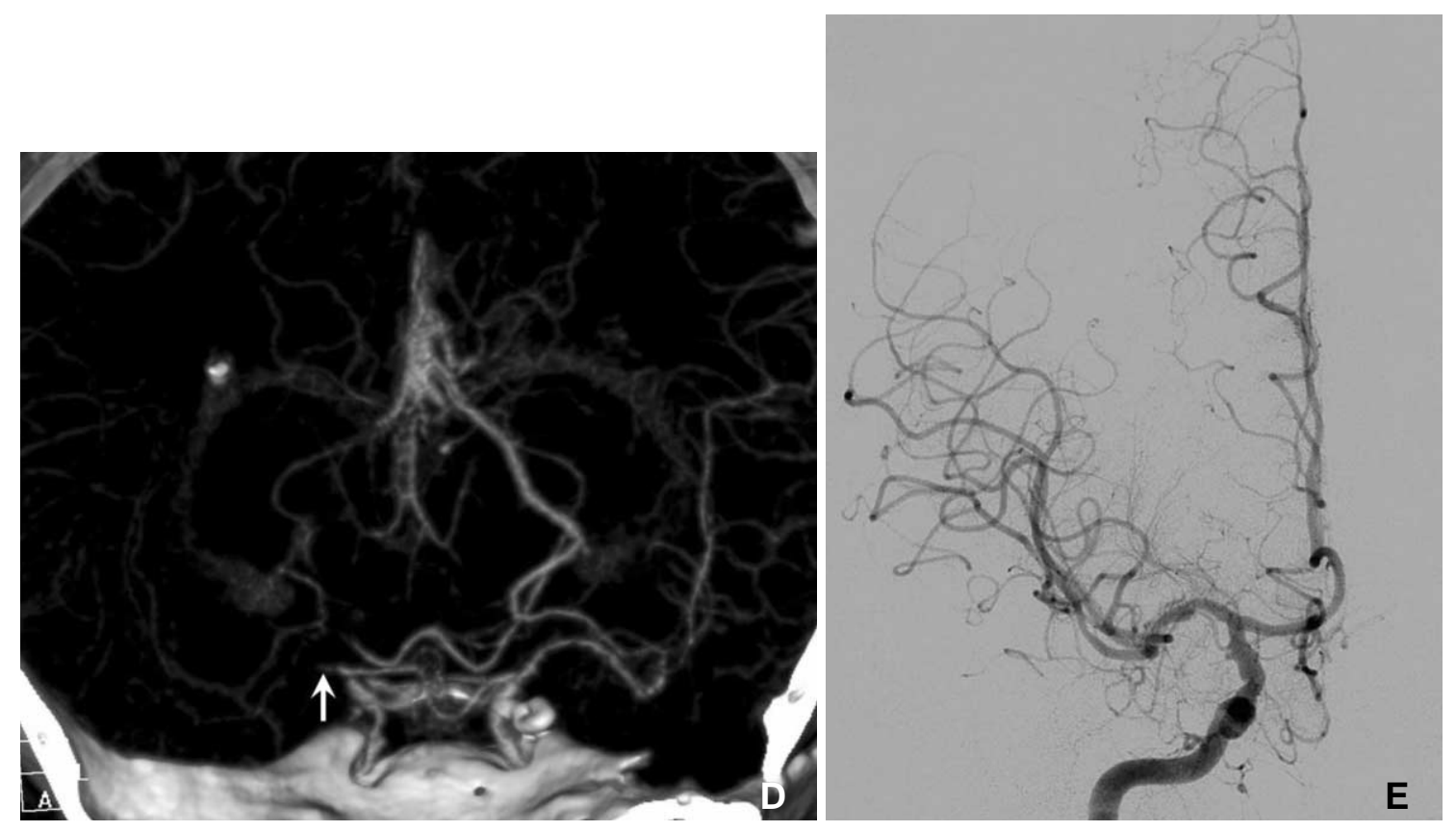

Fig. 1. D. CT angiography that is performed two months later shows persistent occlusion of right MCA (arrow).

E. Twenty months later, right carotid angiography shows the recanalization of previously occluded segment of right MCA.

right middle cerebral artery territory and the vascular reserve was also decreased mildly after acetazolamide injection (Fig. 1C). An echocardiogram and myocardial SPECT excluded cardiac embolus as the etiology of the occlusion. Although the patient had no past medical history, untreated hypertension and diabetic mellitus were found during evaluation. In this setting of presumably chronic occlusion, surgical, endovascular and thrombolytic therapies would cause serious complications. Therefore conservative managements were selected for this patient. The patient was initially treated with antiplatelet and circulating drug and carefully observed for two weeks. He had no further neurologic symptoms.

After two months, the left P-com aneurysm was managed by the microsurgical aneurysmal neck clipping and there were no developing or new neurologic symptoms after surgery. Immediate postsurgical computed tomography angiogram (CTA) showed persistent right MCA occlusion and complete aneurysm clipping (Fig. 1D). The medications were continued during the follow-up period. The brain CTA of 21 months after neck clipping developed recanalization of the previously occluded right MCA. A subsequent conventional angiogram confirmed nearly complete patency of the right MCA with focal mild stenosis, normal blood flow through MCA, and the normalization of the shift of watershed zone (Fig. 1E). Our patient experienced late spontaneous recanaliza- tion and restoration of blood flow by an unknown mechanism within 21 months, without any neurologic deficit.

\section{DISCUSSION}

Spontaneous recanalization of the occluded MCA has been a common finding in acute ischemic stroke. Several studies demonstrated that most recanalization may occur in the acute phase of stroke, within approximately 48 hours after onset $[2,5]$. A recent metaanalysis of thrombolytic therapy attempted to quantify spontaneous recanalization in ischemic stroke. Spontaneous recanalization occurred in $21.4 \%$ of patients within 24 hours and in $52.7 \%$ of patients by a week [6]. Although the analysis of these data had limitations, including the variety of times examined, but the suggestion is that the natural history of cerebral embolus is dissolution and spontaneous recanalization over a period of time. Like these studies, spontaneous recanalization of MCA, in which occlusion is apparent in the acute phase have occasionally occurred in the subacute phase of stroke. But late recanalization in the chronic phase has not been reported to the best of our knowledge.

Unlike recanalization of occluded artery in acute stroke, the spontaneous recanalization of a longstanding occlusion of extracranial artery has been only anecdotally reported [7-9]. The mechanism by which 


\section{Cheol-Young Lee, et al.}

recanalization of chronically occluded carotid arteries occurs is still little known. Lammie et al. [7], in 1999, suggested the possibility of occlusions resulting from ulcerated plaque thrombosis presenting long-term recanalization by thrombolysis. Colon et al. [8], in 1999, published a series of four cases of spontaneous recanalization of the internal carotid artery, which through imaging examinations and intraoperative finding, proved to be a hypertrophy of vasa vasorum, causing reperfusion of the distal to the occlusion. Another possible mechanism is that, in the case of myointimal hyperplasia or atherosclerotic disease, the neovascularization is induced, which in the long term can allow perfusion distal to vessel occlusion. Persistence of some embryonic vessels can also account for the complete non-occlusion of the whole internal carotid artery segment, allowing action of varied mechanisms for vessel recanalization. Nowadays some groups have been following patients with carotid occlusions, with the aim of better evaluating the natural history of such lesions. Verlato et al. [10], in 2000, published a cohort study including 41 patients with carotid occlusion. The mean follow up periods were 44.5 months. In one case with asymptomatic carotid occlusion of 41 patients, spontaneous recanalization was identified at three years after the diagnosis, remaining without symptoms for the whole period.

The mechanism of late recanalization of chronic occlusions of MCA could be one as remarked above or not. Unlike the extracranial carotid artery, the intradural cerebral artery has a little chance to the hypertrophy of vasa vasorum or the revascularization within a plaque due to the nature of intracranial artery, including the lack of vasa vasorum and the smaller caliber. In our case, we cautiously speculate that the recanalization process could be that the repeated embolic formation and accumulation were progressed to complete obstruction for some period of time in focal thrombotic area. After antiplatelet therapy, the thrombolysis occurred gradually instead of repeated embolic accumulation. Finally, spontaneous recanalization occurred at this late stage.

The angiographic changes in our patient illustrate that the chronic MCA occlusion were spontaneously recanalized by an unknown mechanism within 21 months, with a good clinical outcome. The incidence, mechanism, and ideal management of this extraordinary finding remain unclear. Through this rare case, further investigation and studies into the underlying mechanism of spontaneous recanalization should be performed.

\section{References}

1. Klonaris C, Alexandrou A, Katsargyris A, Liasis N, Bastounis E. Late spontaneous recanalization of acute internal carotid artery occlusion. J Vasc Surg 2006;43:844-847

2. Fieschi C, Bozzao L. Transient embolic occlusion of the middle cerebral and internal carotid arteries in cerebral apoplexy. $J$ Neurol Neurosurg Psychiatry 1969;32:236-240

3. Kassem-Moussa H, Graffagnino C. Nonocclusion and spontaneous recanalization rates in acute ischemic stroke: a review of cerebral angiography studies. Arch Neurol 2002;59:1870-1873

4. Oster JM, Aggarwal P. Spontaneous recanalization of the basilar artery with conservative management months after symptom onset. Neurol Int 2009 Nov 16;1:e17

5. Zanette EM, Roberti C, Mancini G, Pozzilli C, Bragoni M, Toni D. Spontaneous middle cerebral artery reperfusion in ischemic stroke. A follow-up study with transcranial Doppler. Stroke 1995;26:430-433

6. Rha JH, Saver JL. The impact of recanalization on ischemic stroke outcome: a meta-analysis. Stroke 2007;38:967-973

7. Lammie GA, Sandercock PA, Dennis MS. Recently occluded intracranial and extracranial carotid arteries. Relevance of the unstable atherosclerotic plaque. Stroke 1999;30:1319-1325

8. Colon GP, Deveikis JP, Dickinson LD. Revascularization of occluded internal carotid arteries by hypertrophied vasa vasorum: report of four cases. Neurosurgery 1999;45:634-637

9. Buslovich S, Hines GL. Spontaneous recanalization of chronic internal carotid artery occlusions: report of 3 cases. Vasc Endovascular Surg 2011;45:93-97

10. Verlato F, Camporese G, Bernardi E, Salmistraro G, Rocco S, Mayellaro V, et al. Clinical outcome of patients with internal carotid artery occlusion: a prospective follow-up study. J Vasc Surg 2000;32:293-298 